\title{
A Laser With a Multihole Diaphragm
}

\author{
Tadashi Morokuma*
}

\author{
(October 21, 1963)
}

\begin{abstract}
The properties of a laser with a multihole diaphragm were both theoretically and experimentally examined. This laser may be called a multibeam laser. Laser action was observed in the optical paths which were defined by the position of the holes and the cavity configuration. Interference fringes were observed on one of the cavity mirrors. A wavelength dependent interaction among the beams was observed. It is beileved that the wavelength of a beam can be stabilized by the intensities of the other beams. A possible method will be proposed for the stabilization.
\end{abstract}

\section{Introduction}

In order to use continuous wave lasers for interferometric length measurement, their wavelengths should be stable enough to secure the required accuracy. The stability of a helium-neon laser has been intensively studied by Javan et al., [1, 2]. ${ }^{1}$ They have obtained short-term stability to better than two parts in $10^{13}$ by isolating their lasers from mechanical vibration, and, they were able to reset the wavelength with an accuracy of one part in $10^{9}$. Gould [3] proposed to use the pulling effect for stabilization. A different approach has been pursued in this laboratory by placing a multihole diaphragm inside the confocal cavity of a laser.

The interesting phenomena found in the beams of the laser and also a possible method for stabilization will be described. The laser is provided with a diaphragm containing five circular holes; four of them are symmetrically located around a central one. The diaphragm is placed inside the cavity and in front of the one of the cavity mirrors. It is possible to obtain laser action so that one beam travels back and forth through the central hole on the axis of the cavity. The other two beams travel diagonally through the top and bottom holes, and the two side holes, respectively. Five bright round spots have been observed on the mirror that is covered with the diaphragm and a larger spot with interference fringes on the other mirror.

This laser essentially consists of three sublasers with common cavity mirrors. Their wavelengths are determined by the effective cavity spacings corresponding to the optical paths for one transit of each beam in the cavity. According to the resonance condition of a confocal cavity [4], it is possible for the oscillation in each beam to take place at several

*Guest worker-Vational Bureau of Standards, present address: Olympus Optical Company Ltd. Tokyo, Japan.

Figures in brackets indicate the literature references at the end of this paper. discrete wavelengths separated by a mode spacing of the cavity. Fabry-Perot rings, however, show interaction among the beams so that the oscillation at certain wavelengths is suppressed. Therefore, the wavelength relation is more complicated than expected.

It was possible for the laser to satisfy the necessary conditions for the wavelength stabilization by the use of a suitable diaphragm, and by the adjustment of its position and the excitation power.

\section{Modes of Oscillation}

The 1-m laser used in this laboratory is composed of an $80-\mathrm{cm}$ discharge tube and a multihole diaphragm that is located close to one of the cavity mirrors. The discharge tube contains a mixture of $1 \mathrm{~mm} \mathrm{Hg}$ of helium and $0.1 \mathrm{~mm} \mathrm{Hg}$ of neon. The maximum gain of the tube is about 5 percent per pass at $1.152 \mu$.

The schematic diagram of the laser and a photograph of the diaphragm are shown in figures $1 \mathrm{a}$ and $\mathrm{b}$. The beam is elliptical in shape, approximately 10 $\mathrm{mm}$ along one axis and $8 \mathrm{~mm}$ along the other. This distortion is due to an aberration in the Brewster's angle windows. The central hole was purposely made smaller than the outer holes so that the larger diffraction loss due to the smaller opening would compensate for the larger gain of the central beam. Thus, each of the three beams can be operated in a single mode with the intensities almost equal to each other. The diameter of the holes and spacings should be determined by several factors, such as the gain, the diameter of a discharge tube, the aberration of windows or mirrors, etc. The modes of oscillations are shown schematically in figure 2. The frequencies of the beams are given by

$$
f_{\circ}=\frac{c N_{o}}{2 d_{0}}
$$

for beam 1

$$
f_{1}=\frac{c N_{1}}{4 d_{1}}
$$




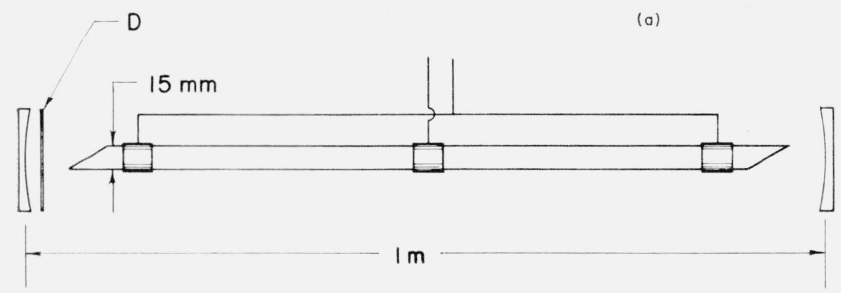

Figure 1a. Schematic diagram of a laser with a multihole diaphragm, D.

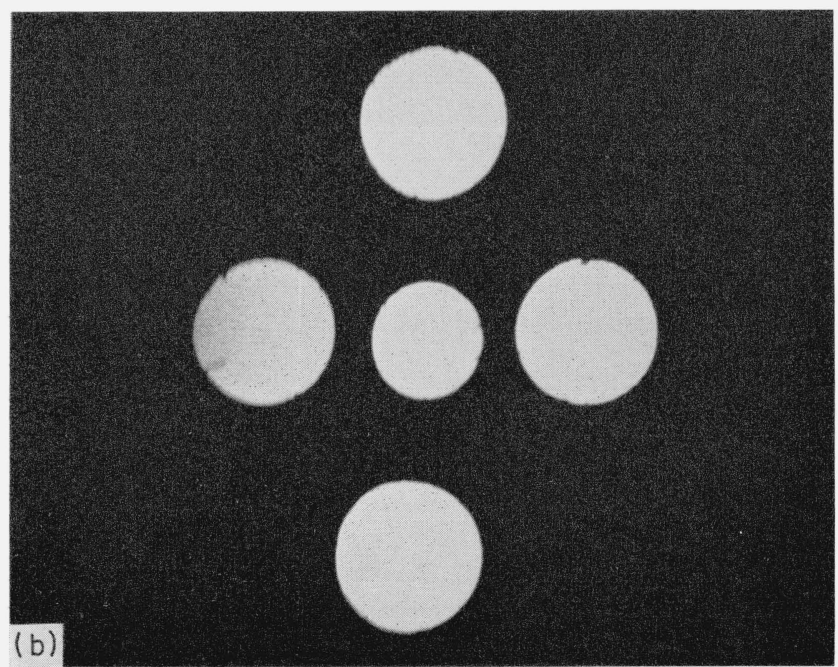

Figure 1b. Photograph of the diaphragm.

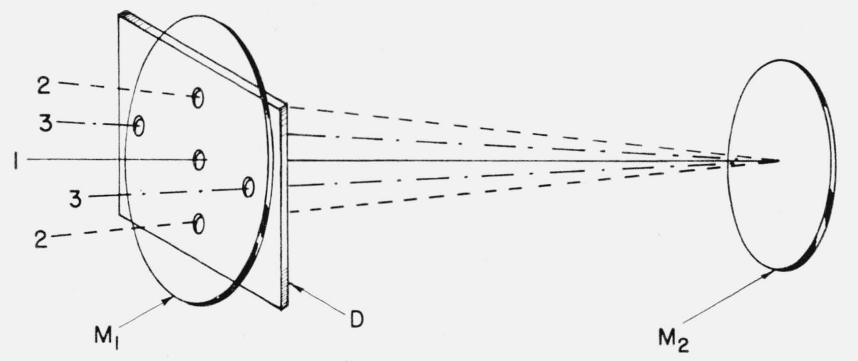

$M_{1}, M_{2}$ MIRRORS

D DIAPHRAGM

I. - CENTRAL BEAM

2.--- TOP \& BOTTOM BEAM

3. - - SIDE BEAM

Figure 2. Modes of oscillation. for beam 2

for beam 3

$$
f_{2}=\frac{c N_{2}}{4 d_{2}}
$$

where $N_{o}, N_{1}$, and $N_{2}$ are order numbers (integers), $d_{0}, d_{1}$, and $d_{2}$ are optical paths between the cavity mirrors, and $c$ is the velocity of light.

The modes of outer beams 2 and 3 will be called even modes when $N_{1}$ and $N_{2}$ are even and odd modes when they are odd. In case of an ideal confocal cavity, the optical paths $d_{0}, d_{1}$, and $d_{2}$ are all the same so that $f_{1}$ and $f_{2}$ are equal and the frequencies of the even modes of the outer beams are equal to those of the central beam. If there is an aberration withm the cavity, however, the three frequencies may be slightly different from each other. This will play an important role in the discussion of the wavelength relation, as is described later.

\section{Mode Pattern}

Since a diaphragm is placed in front of only one of the cavity mirrors, symmetrical intensity distribution, as described in Fox and Li's theory, [5] cannot be expected. In other words, five bright spots are observed on the same mirror, and a large spot with interference patterns is observed on the other mirror. The five spots are shown in figure 3 . The interference patterns are shown in figures 6,7 , and 8 .

\subsection{Central Beam}

Figure 4 shows the beam on mirror $\mathrm{M}_{2}$ when the outer holes are blocked. This photograph was taken with a low excitation power so that higher order

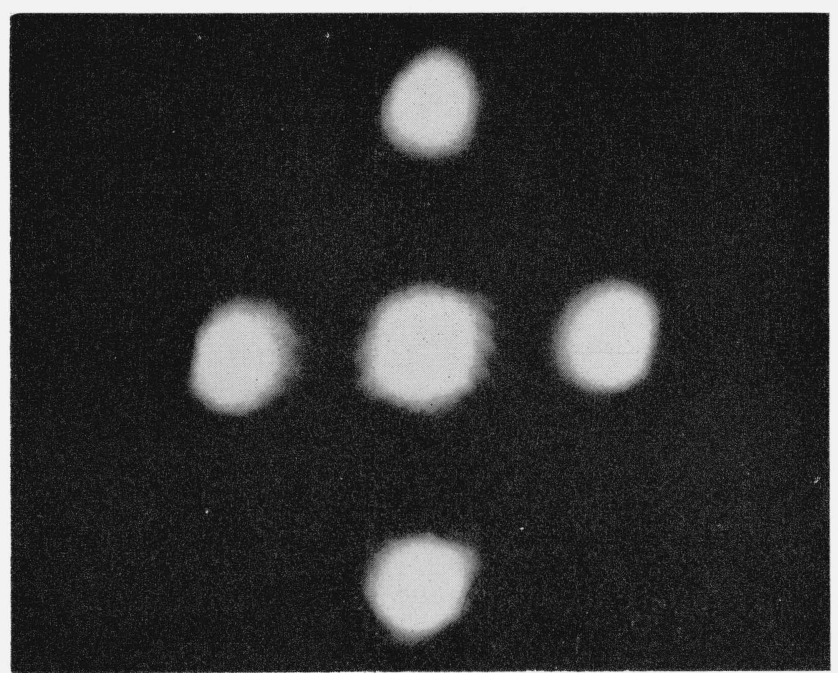

Figure 3. Cross section of the beams on mirror $M_{1}$, with diaphragm as shown in figure 2.

2 The patterns which are seen in the photograph are produced in the image converter used in this experiment. They are not inherent in the beam. 


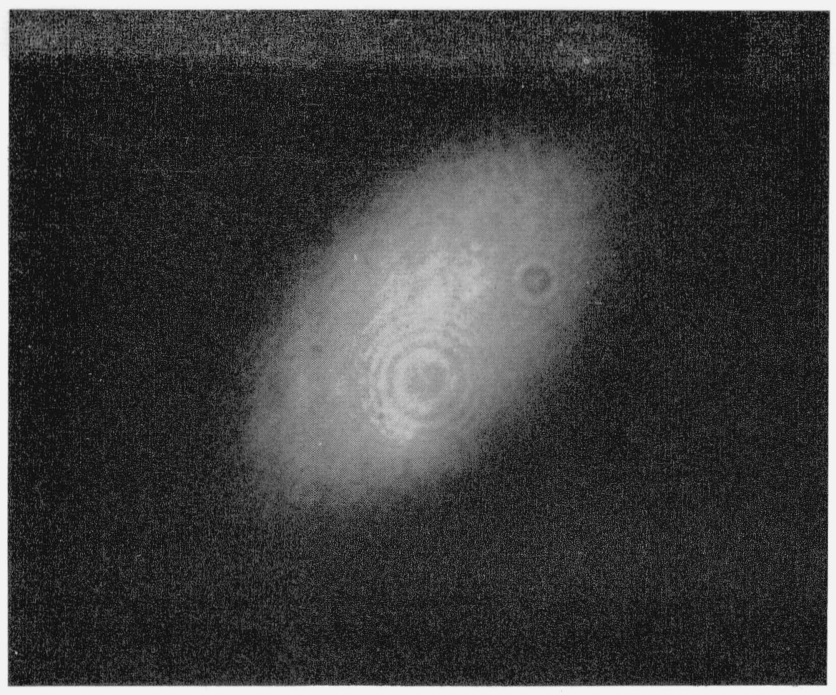

Figure 4. Cross section of the central beam on mirror $M_{2}$, with diaphragm as shown in figure 5.

modes did not appear. The cross section of the elliptical beam is about $4.8 \mathrm{~mm}$ along one axis and $2.9 \mathrm{~mm}$ along the other. This cross section is much larger than that of the central beam in figure 3, which measures $0.9 \mathrm{~mm}$ in diameter. In figure 4 , of course, no fringe pattern is observed. ${ }^{2}$

These spot sizes can be calculated with a theory similar to Fox and Li's [5].

\subsection{Outer Beams}

Consideration will be given first to the case of two holes and then to the case of four holes. The observed fringe patterns can be explained as the result of interference between two or four spherical wave fronts.

\section{a. Two Holes}

When the central and the two side holes are blocked, two beams are observed coming out of the laser in different directions, as shown in figure 5 . Figure 6 shows interference fringes observed on $\mathrm{M}_{2}$ when the excitation power is decreased until single mode operation is achieved. The spacing, $s$, of the fringes is given by $s=d \lambda / a$, where $a$ is the distance between the holes, $d$ the mirror separation and $\lambda$ the wavelength. In the case $d=1 \mathrm{~m}, \lambda=1.15 \mu$, and $a=3.1 \mathrm{~mm}$, the spacing is $0.37 \mathrm{~mm}$. This agrees with the spacing measured on figure 6 . It is to be noted that a bright fringe is observed on the axis of the cavity for an even mode and a dark fringe for an odd mode. Since the phase difference between the two beams is $\pi N_{1}$ on mirror $\mathrm{M}_{2}$ from eq (2), it becomes $2 n \pi$ radians for an even mode $\left(N_{1}=\right.$ even $)$ and $(2 n+1) \pi$ radians for an odd mode $\left(N_{1}=\right.$ odd $)$. In this case $n$ is an integer. These two fringe patterns intermesh with each other. Consequently, no fringes will be observed when even and odd modes are coexistent and their intensities are exactly equal. Figure 7 shows the intermeshed fringe patterns observed in the case of multimode operation.

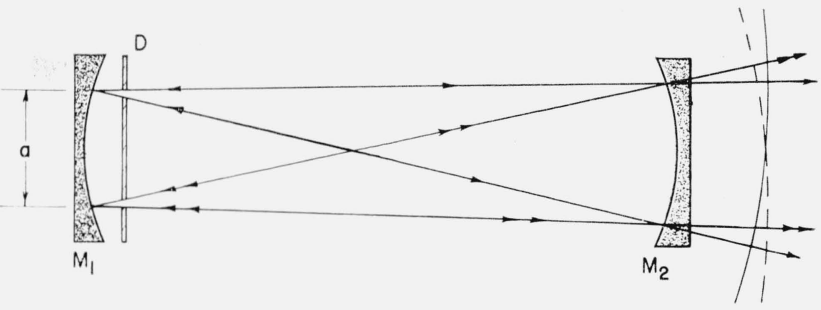

Figure 5. Optics of a two-beam laser.

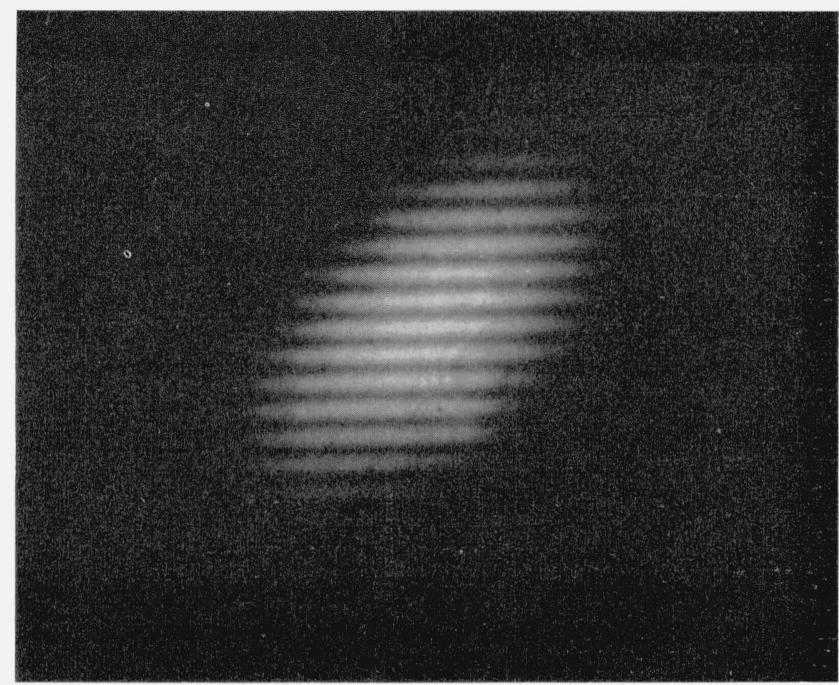

Figure 6. Fringes observed on mirror $M_{2}$, with diaphragm as shown in figure 5.

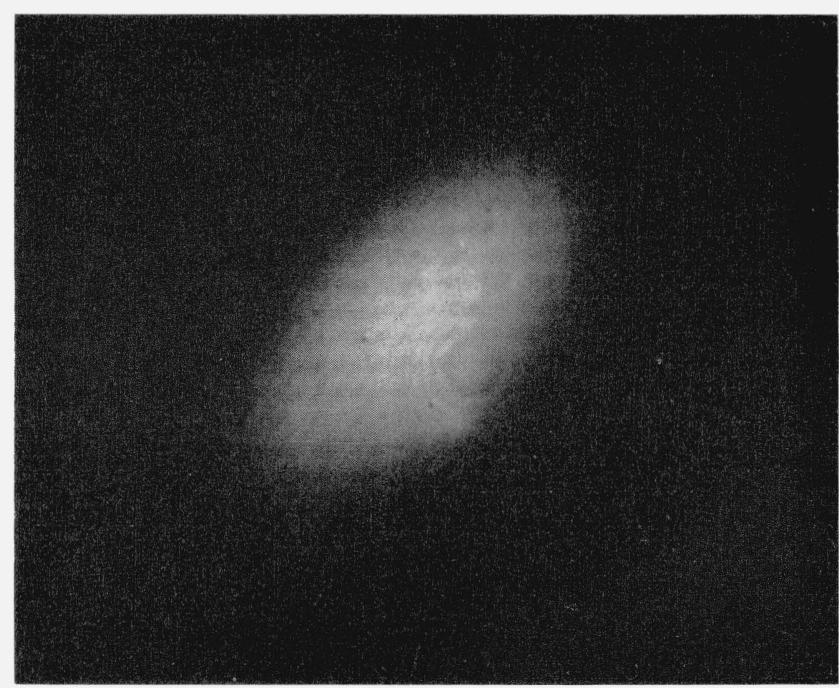

FiguRE 7. Intermeshed fringe patterns. 

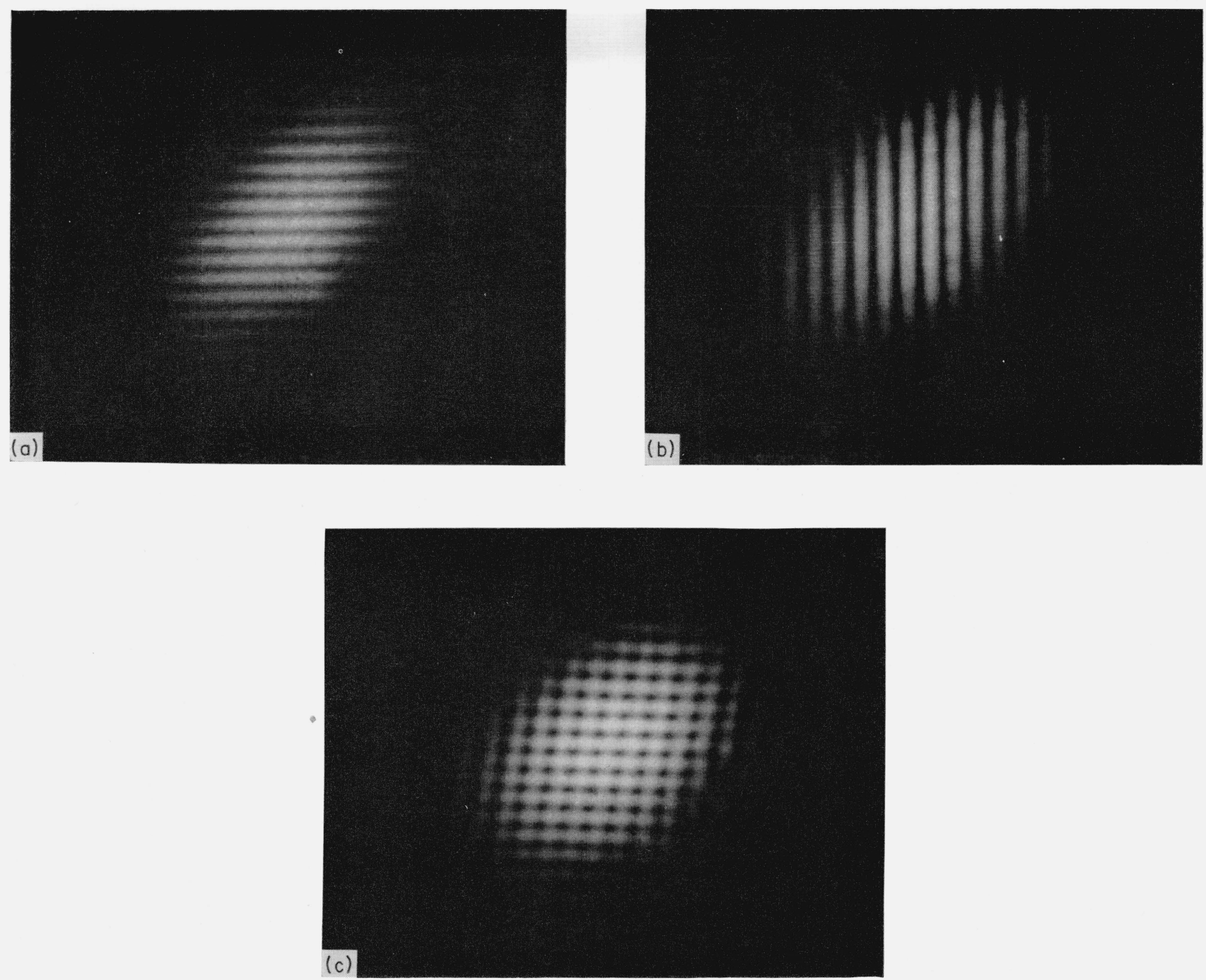

Figure 8. Fringes observed on mirror $M_{2}$, with diaphragm as shown in figure 9.

(a) Fringes between beams 1 and 2,

(b) fringes between beams 3 and 4 ,

(c) superposition of (a) upon (b).

b. Four Holes-The Superposition and the Interference of Two Sets of Fringes

One of the interference patterns is shown in figure $8 \mathrm{c}$. It is obtained by superimposing the iringe pattern of figure $8 \mathrm{a}$ on the one of figure $8 \mathrm{~b}$. The pattern in figure $8 \mathrm{a}$ is the same as that in figure 6 . Another pattern that has been observed is composed of bright spots. This pattern is very unstable and remains stationary for only a few seconds. (See the discussion pertaining to figure 11.) The intensity distribution of these patterns can be easily explained by assuming that the wave fronts of the four beams are replaced by plane wave fronts propagating in the directions connecting the center of curvature of mirror $\mathrm{M}_{1}$ and the centers of the holes, as shown in figure 9 . In the $x-y$ plane, which is tangential to mirror $\mathrm{M}_{2}$, with the origin of the coordinates on the optical axis, the four beams can be represented in the following forms:

$$
\begin{aligned}
& A_{1}=\exp i\left(\omega_{1} t-k_{1} a x / 2 d_{1}\right) \\
& A_{2}=\exp i\left(\omega_{1} t+k_{1} a x / 2 d_{1}+\alpha\right) \\
& A_{3}=C \exp i\left(\omega_{2} t-k_{2} b y / 2 d_{2}+\gamma\right) \\
& A_{4}=C \exp i\left(\omega_{2} t+k_{2} b y / 2 d_{2}+\gamma+\beta\right)
\end{aligned}
$$

for beam 1 .

for beam 2 .

for beam 3 .

for beam 4 .

Here $k_{1}$ and $k_{2}$ are given by $k_{1}=2 \pi / \lambda_{1}$ and $k_{2}=2 \pi / \lambda_{2}$ where $\lambda_{1}$ and $\lambda_{2}$ are wa velengths; $\omega_{1}$ and $\omega_{2}$ are angular frequencies; $a$ and $b$, the spacings between holes; $\alpha$ and $\beta$, the phase differences between beams 1 and 2 , and beams 3 and 4 , respectively. $C$ is the amplitude ratio between $A_{1}$ and $A_{3}$. The origin of time, $t$, is 


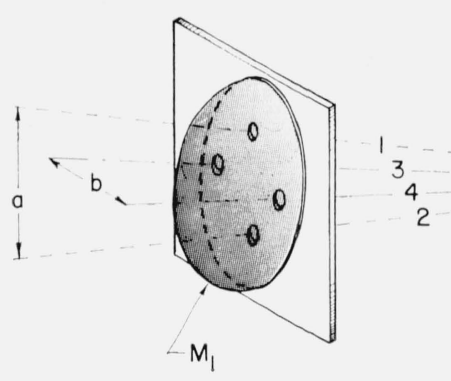

Figure 9. Optics of a four-beam laser.

chosen so that the phase angle of beam 1 is aqual to zero at $x=0$. The initial phase angle of beam 3 is then given by $\gamma$ at $y=0$. It is to be noted that $\alpha$ and $\beta$ should be $2 n \pi$ or $(2 n+1) \pi$ radians, as previously described, corresponding to even or odd modes. The intensity distribution is

$I(x, y) \propto\left\{\cos ^{2}\left(k_{1} a x / 2 d_{1}+\alpha / 2\right)\right.$

$$
\left.+C^{2} \cos ^{2}\left(k_{2} b y / 2 d_{2}+\beta / 2\right)\right\}
$$

when $\lambda_{1} \neq \lambda_{2}$, and

$$
\begin{aligned}
I(x, y) \propto & \left\{\cos ^{2}\left(k a x / 2 d_{1}+\alpha / 2\right)\right. \\
& +C^{2} \cos ^{2}\left(k b y / 2 d_{2}+\beta / 2\right) \\
+ & 2 C \cos \left(k a x / 2 d_{1}+\alpha / 2\right) \cos \left(k b y / 2 d_{2}\right. \\
& +\beta / 2) \cos (\alpha / 2-\beta / 2-\gamma)\}
\end{aligned}
$$

when $\lambda_{1}=\lambda_{2}=\lambda$, and $l=2 \pi / \lambda$. Spacings $d_{1}$ and $d_{2}$ are almost equal to the spacing $d_{0}$ on the axis and the difference is less than $\lambda / 2$. Therefore, they can be replaced by $d_{0}$ in the above equations. Then

$$
\begin{aligned}
I(x, y) \propto\left\{\operatorname { c o s } ^ { 2 } \left(k_{1} a x / 2 d_{0}\right.\right. & +\alpha / 2) \\
& \left.+C^{2} \cos ^{2}\left(k_{2} b y / 2 d_{0}+\beta / 2\right)\right\}
\end{aligned}
$$

when $\lambda_{1} \neq \lambda_{2}$ and

$$
\begin{aligned}
I(x, y) \propto & \left\{\cos ^{2}\left(k a x / 2 d_{0}+\alpha / 2\right)\right. \\
& +C^{2} \cos ^{2}\left(k b y / 2 d_{0}+\beta / 2\right) \\
& +2 C \cos \left(k a x / 2 d_{0}+\alpha / 2\right) \cos \left(k b y / 2 d_{0}\right. \\
& +\beta / 2) \cos (\alpha / 2-\beta / 2-\gamma)\}
\end{aligned}
$$

when $\lambda_{1}=\lambda_{2}=\lambda$. Figure 10 shows the intensity distribution calculated from eq (4) for $\lambda=1.152 \mu$, $d=1 \mathrm{~m}, a=3.1 \mathrm{~mm}, b=4.1 \mathrm{~mm}$, and $C=1$. The $\max -$ imum intensity is normalized to 1.0 in the drawing. This is the case where the two patterns are simply overlapped without interference between them.

When $\lambda_{1}$ is exactly equal to $\lambda_{2}$, two kinds of patterns have been observed. One pattern has the same distribution as shown in figure 10 and is given by eq (5) when $\cos (\alpha / 2-\beta / 2-\gamma)=0$. The theoretical distribution of another pattern is shown in

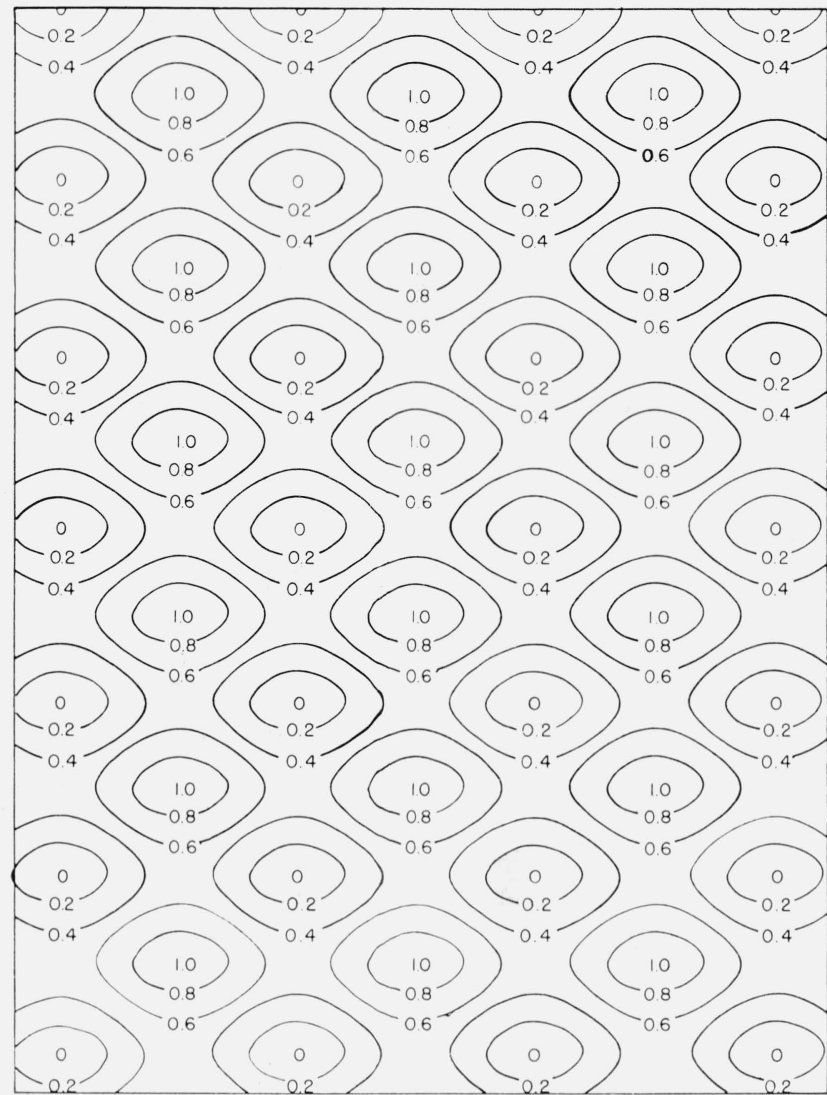

Figure 10. Theoretical intensity distribution of fringes when $\lambda_{1} \neq \lambda_{2}$ or $\cos (\alpha / 2-\beta / 2-\gamma)=0$ at $\lambda_{1}=\lambda_{2}$.

figure 11. This pattern is composed of bright spots and dark lines. They are obtained when $\cos (\alpha / 2$ $-\beta / 2-\gamma)= \pm 1$ in eq $(5)$.

A possible explanation for this is as follows: In the volume that beams occupy in common, they interact with each other. In other words, the photon in one pair of beams stimulate the laser oscillation in another pair of beams. Therefore, the phase difference would be zero, on the assumption that stimulated radiation is in phase with the incident radiation. When both pairs of beams are either in even or in odd modes, the cosine term is equal to unity; that is, $\cos (\alpha / 2-\beta / 2-\gamma)= \pm 1$. If one pair of beams is in an even mode and another pair in an odd mode, the term vanishes; that is, $\cos (\alpha / 2-\beta / 2-\gamma)=0$ The experimental results are not sufficient to prove the validity of the above statements and further investigation is necessary.

\section{Wavelength Relation}

A wavelength dependent interaction of one beam on the others has been found by means of a FabryPerot etalon. Although the diameter of the central hole was purposely made smaller than the outer holes, the central beam is more intense than the other beams. The even modes of the outer beams 


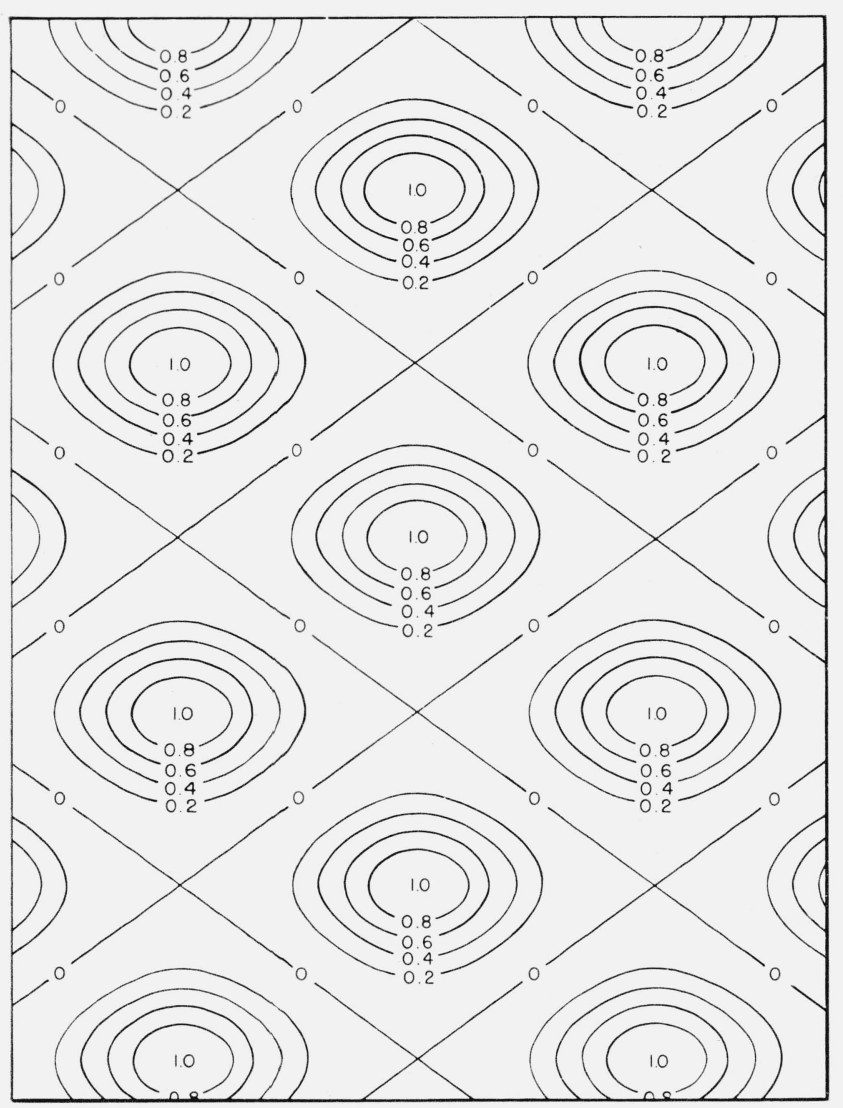

Figure 11. Theoretical intensity distribution of fringes when $\lambda_{1}=\lambda_{2}$ and $\cos (\alpha / 2-\beta / 2-\gamma)=1$.

could not be observed at low excitation power. Since every beam passes a common space at one end of the cavity, the gain for the modes becomes lower than the threshold due to the high density of photons in the central beam. Hence at low excitation level, it is possible for outer beams to have only odd modes separated by $150 \mathrm{Mc} / \mathrm{s}$ for a $1-\mathrm{m}$ confocal cavity. Also interaction between outer beams results in the suppression of some odd modes in one beam by another.

\subsection{Experimental Arrangement}

In order to study the wavelength relation among the beams, Fabry-Perot etalons are used as shown in figure 12. The first lens $L_{1}$, is focused on mirror $\mathrm{M}_{2}$. A spherical wave front from a point source on $\mathrm{M}_{2}$ becomes a plane wave front, passing through either a $1-\mathrm{m}$ or a $50-\mathrm{cm}$ Fabry-Perot etalon, then through the second lens, $L_{2}$. Haidinger fringes are formed on its focal plane, and the surface of mirror $\mathrm{M}_{2}$ is also imaged on the same plane. Therefore, the Haidinger fringes are modulated by the image of a pattern on $\mathrm{M}_{2}$. A given set of fringes identifies the location of the beams, either by a visual or a photographic method. For example, if a set of fringes is composed of continuous rings with even

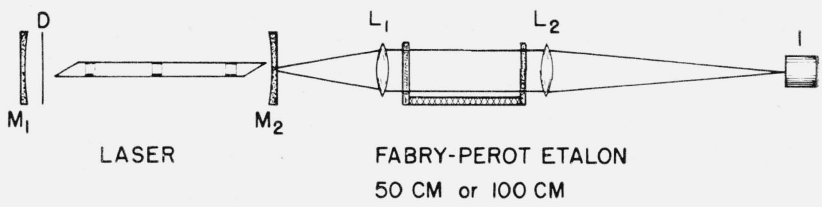

$$
\begin{aligned}
& L_{1}: f=30 \mathrm{~cm} \quad L_{2}: f=130 \mathrm{~cm} \\
& 1: \text { IMAGE CONVERTER }
\end{aligned}
$$

Figure 12. Optical arrangement to study the wavelength relation.

distribution of intensity, these fringes are formed by the central beam. If fringes are broken vertically with dark parallel lines equally spaced, these fringes are formed by the side beams.

No attempt was made to eliminate the feedback of photons from the etalon to the laser.

\subsection{Experimental Results}

When a 1-m Fabry-Perot etalon is used, the Haidinger fringes of all the even modes, having approximately the same diameters, appear to be superimposed on each other. Those of the odd modes are similarly superimposed with their bright fringes between the bright fringes of the even modes.

\section{a. Interaction}

When the laser tube is excited with low energy, the central beam can suppress oscillation in the even modes of the outer beams, as previously described. Figure 13a shows fringes obtained when only the top and bottom holes are open. Both even and odd modes are clearly seen in figure 13a, which shows the phase shift equal to $\pi$ radians between them.

If the central hole is opened, without changing other conditions, one set of the broken fringes of even modes changes into a set of continuous fringes but another set remains broken as shown in figure $13 \mathrm{~b}$. This result shows the suppression of oscillation in the even modes of the outer beams by the central beam. In other words, the gain for these modes reduces below the threshold because neon atoms are partly used to emit the photons of the central beam. Interaction between the two pairs of outer beams is not so distinct as is that between the central beam and the outer beam. It may be useful to evalute the intensity of the beams in order to understand the interaction. In case of a single beam laser, intensity is approximately proportional to the value $G-g^{[6]}$. Here $G$ and $g$ are the gain per pass before and after laser action. Therefore, gain $g$ can be written in the form $g=$ $G-h I$, where $h$ is a constant and $I$ the intensity of the beam inside the cavity. In case of a three-beam laser, the following assumption may be made for a simple treatment.

In portion $A$, of the tube in figure 14 , the three beams have common volume and interaction takes place. Beams 2 and 3 are degenerate in frequency and intensity. In portion $B$, they take different paths, therefore no interaction occurs. 

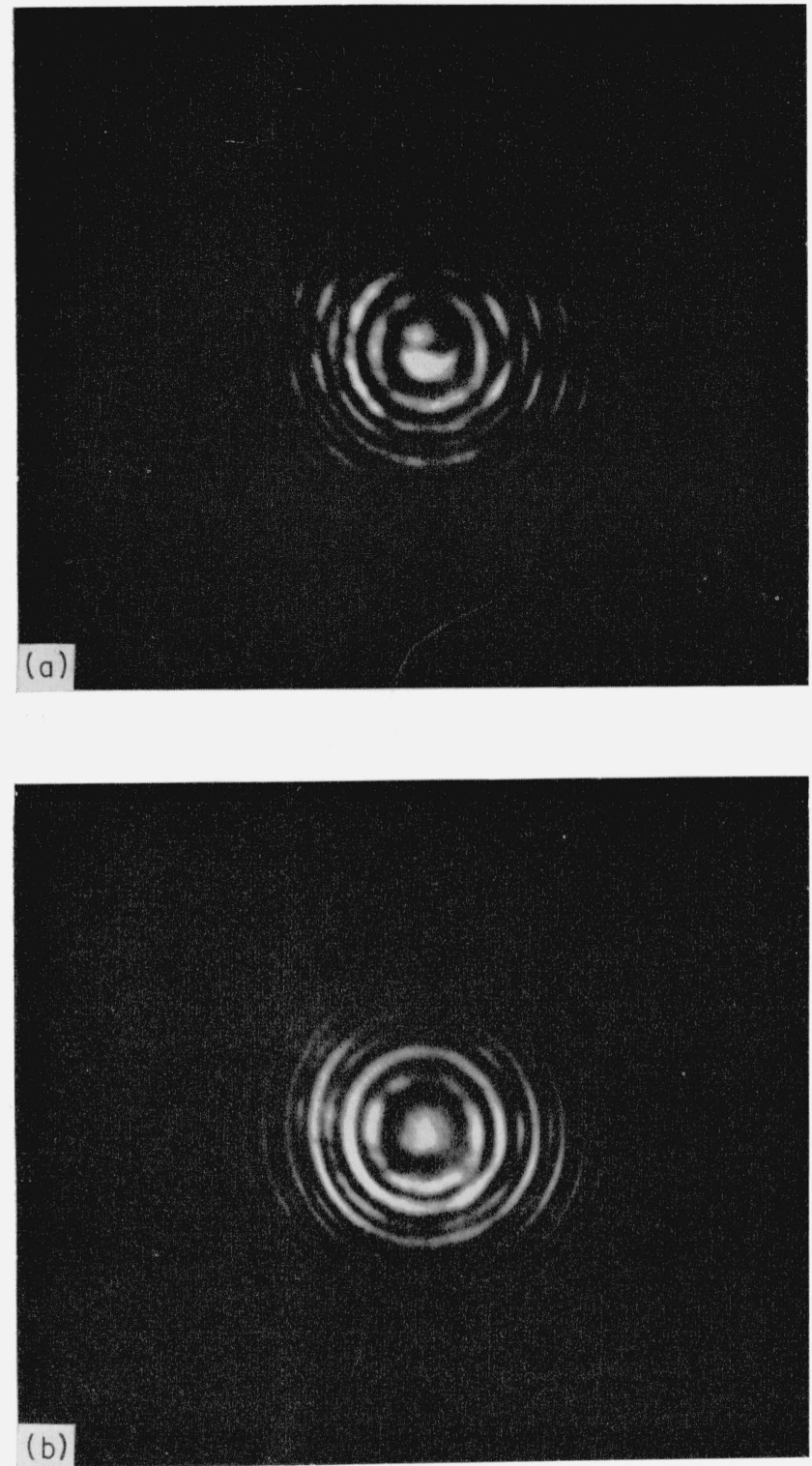

FIguRe 13. Haidinger fringes with a 1-m Fabry-Perot etalon. In (a), the top and bottom holes are open and in (b), the central hole is also open.

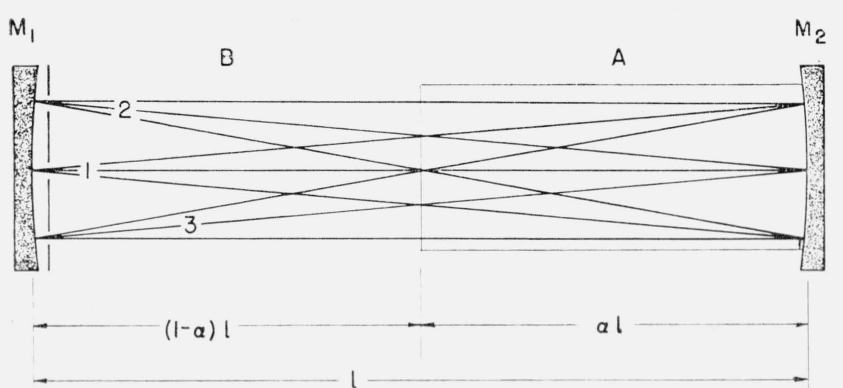

FIgURE 14. Volumes $\mathrm{A}$ and $\mathrm{B}$, with and without interaction of beams, in a three-beam laser.
If the lengths of portions $A$ and $B$ are given by $\alpha l$ and $(1-\alpha) l$, respectively, $l$ being the total length of the tube, the gain for beam 1 is given by

$$
\begin{aligned}
g_{1} & =(1-\alpha)\left(G-h I_{1}\right)+\alpha\left\{G-h\left(I_{1}+2 I_{2}\right)\right\} \\
& =G-h I_{1}-2 \alpha h I_{2} .
\end{aligned}
$$

Here $\alpha$ will be called a coupling constant. Subscript 1 denotes the central beam; subscript 2 denotes the outer beams.

For beams 2 and 3, the gain is given by

$$
g_{2}=G-\alpha h I_{1}-(1+\alpha) h I_{2} .
$$

Now assume that laser oscillation takes place in all of the beams. Gains $g_{1}$ and $g_{2}$ will decrease as intensities $I_{1}$ and $I_{2}$ increase. They will become equal to the total energy losses $L_{1}$ and $L_{2}$ respectively, after the oscillation reaches equilibrium, that is

$$
\begin{array}{ll}
L_{1}=G-h I_{1}-2 \alpha h I_{2} & \text { for beam } 1 \\
L_{2}=G-\alpha h I_{1}-(1+\alpha) h I_{2} & \text { for beams } 2 \text { and } 3 .
\end{array}
$$

Solving the above equations, intensities $I_{1}$ and $I_{2}$ are given by

$$
\begin{aligned}
& I_{1}=\left\{(1+\alpha) J_{1}-2 \alpha J_{2}\right\} /(1-\alpha)(1+2 \alpha) \\
& I_{2}=\left(J_{2}-\alpha J_{1}\right) /(1-\alpha)(1+2 \alpha)
\end{aligned}
$$

where $J_{1}=\left(G-L_{1}\right) / h$, and $J_{2}=\left(G-L_{2}\right) / h$. Since intensity is positive, the following relation should be held

or

$$
1 / \alpha>J_{1} / J_{2}>2 \alpha /(1+\alpha)
$$

$$
1 / \alpha>\left(G-L_{1}\right) /\left(G-L_{2}\right)>2 \alpha /(1+\alpha) \text {. }
$$

When $\left(G-L_{1}\right) /\left(G-L_{2}\right) \geq 1 / \alpha$, the laser action takes place only in the central beam, with the intensity given by

$$
I_{1}=\left(G-L_{1}\right) / h
$$

On the other hand, the action takes place in outer beams 2 and 3 , with the intensity given by

$$
I_{2}=\left(G-L_{2}\right) /(1+\alpha) h
$$

when $\left(G-L_{1}\right) /\left(G-L_{2}\right) \leq 2 \alpha /(1+\alpha)$. Figure 15 shows the criteria given by the above inequalities. Oscillation takes place only in the central beam when $J_{1} / J_{2}$ falls in region (a). It takes place in all the beams when $J_{1} / J_{2}$ falls in region (b), and in beams 2 and 3 when $J_{1} / J_{2}$ falls in region (c).

The value of $\alpha$ is approximately 0.6 for the laser used in this experiment. Therefore, $\left(G-L_{1}\right) /\left(G-L_{2}\right)$ should be larger than 1.67 in order to make $J_{1} / \boldsymbol{J}_{2}$ fall in region (a) and thus suppress oscillation in the outer beams by the central beam. In the case of a five beam laser, similar treatment may be applied by using different values of $\alpha$ for each pair of beams, also by using different values of $J$. If the coupling constants are all the same, the intensities 


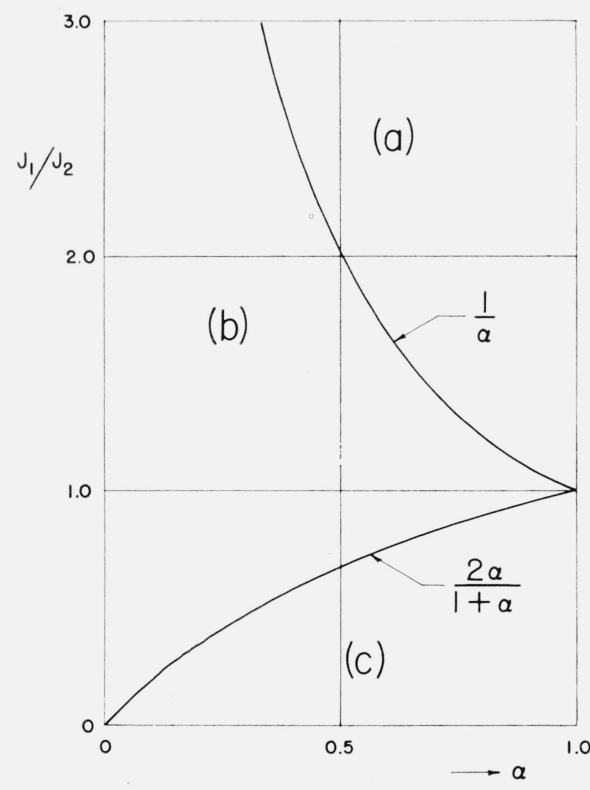

FIGURE 15. Range of $\mathrm{J}_{1} / \mathrm{J}_{2}$ versus coupling constant $\alpha$. In the region (a) only the central beam exists; in (b) the central and all cuter beams exist; and in (c) only the outer beams exist.

of the beams are given by

$$
\begin{aligned}
& I_{1}=\left\{(1+3 \alpha) J_{1}-2 \alpha J_{2}-2 \alpha J_{3}\right\} /(1-\alpha)(1+4 \alpha) \\
& I_{2}=\left\{(2 \alpha+1) J_{2}-2 \alpha J_{3}-\alpha J_{1}\right\} /(1-\alpha)(1+4 \alpha) \\
& I_{3}=\left\{(2 \alpha+1) J_{3}-2 \alpha J_{2}-\alpha J_{1}\right\} /(1-\alpha)(1+4 \alpha) .
\end{aligned}
$$

Here, the subscripts 2 and 3 represent the top and bottom beams and the side beams respectively. From the above equations, the following condition is necessary to suppress the oscillation in the outer beams:

$$
1 / \alpha \leq 2 J_{1} /\left(J_{2}+J_{3}\right) .
$$

\section{b. Wavelength Relation}

It is possible to adjust a five-beam laser so that single mode operation is achieved in each beam when the wavelength of the central beam lies approximately at the center of Doppler distribution. Figure 16 shows the Haidinger fringes obtained with a $50-\mathrm{cm}$ Fabry-Perot etalon arranged similarly to the optical system in figure 12 . The wavelengths are all different and the central beam is located between the outer beams. The wavelength separation between adjacent fringes is found to be approximately $2.5 \times 10^{-3} \mathrm{~cm}^{-1}$, equal to the mode separation of the outer beams. Figure 17 shows the mode relation between beams and the gain distribution of the laser tube with respect to wavelength, $\lambda$.

$L_{1}$ and $L_{2}$ are, respectively, the total energy losses in the central and outer beams. In the central region, $A$, the net gain, $G-L_{1}$, of the central beam is much higher than those of the outer beams, so that oscillation cannot take place in these beams. In the region, $B$, no mode exists in the central beam and interaction is observed only between the outer

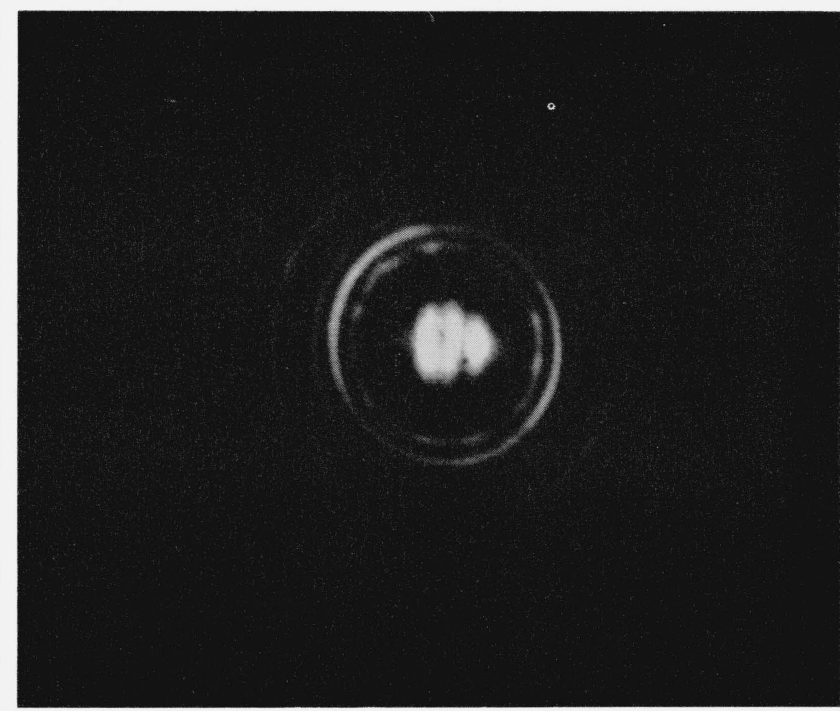

FIGURE 16. Haidinger fringes showing single-mode operation in each beam.

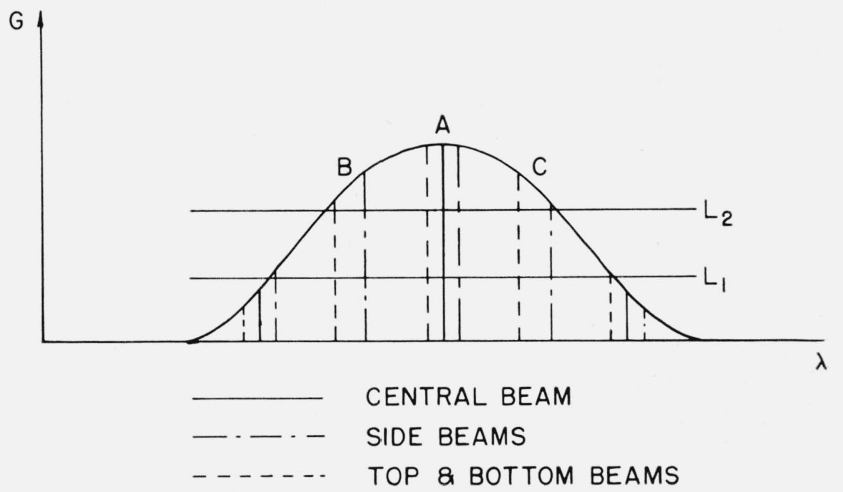

FIGURE 17. Mode relation and gain distribution with respect to the wavelength corresponding to the fringes in figure 16.

The region $A$ corresponds to the mode of the central beam and the even modes of the outer beams. The regions $B$ and $C$ correspond to the odd modes of the outer beams.

beams. If $J_{3} / J_{2} \geq\left(\alpha_{23}+1\right) / 2 \alpha_{23}$, the oscillation in the top and bottom beams is suppressed by the side beams. Here $J_{2}$ and $J_{3}$ are defined in the same way as in the previous section and $\alpha_{23}$ is the coupling constant between the two outer beams. The situation is reversed in the region $C$ and oscillation takes place only in the top and bottom beams. When the effective cavity spacing changes due to, for example, the heat developed inside the tube, the wavelength relation changes as shown in figure 18. Here two modes are seen in the central beam and the modes of the outer beams lie in between, with approximately the same wavelength. The wavelength relation between these modes and the gain distribution are shown in figure 19 . In region $A$, the ratio of $J_{3} / J_{2}$ is nearly equal to unity, so that both modes exist. In regions $B$ and $C$ only the central beam exists. 


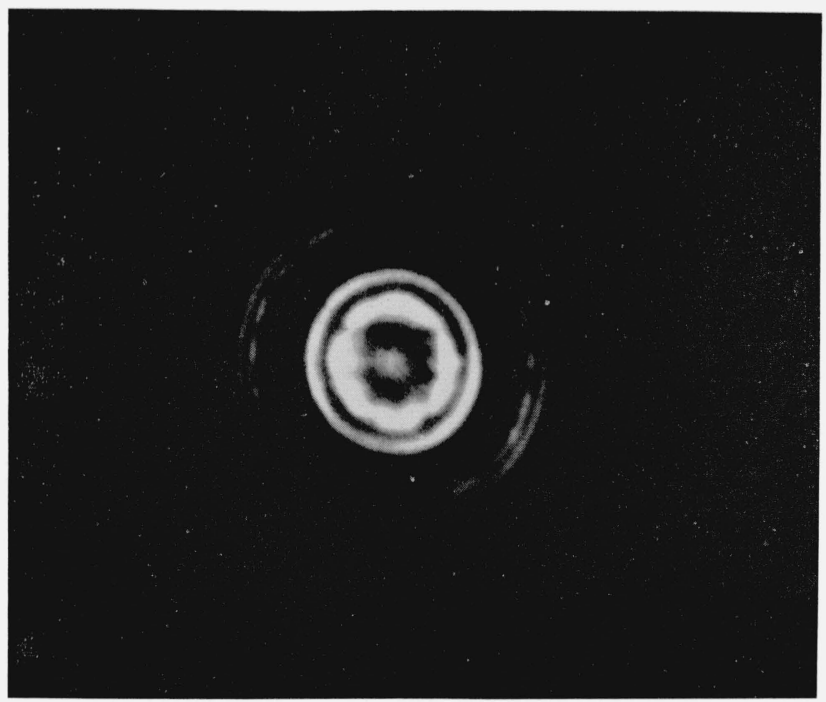

Figure 18. Haidinger fringes showing two-mode operation in the central beam.

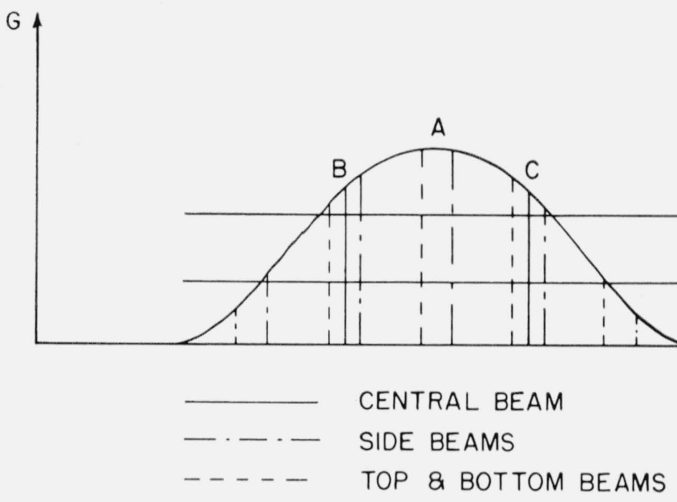

FIGURE 19. Mode relation and gain distribution corresponding to fringes in figure 18.

The region $A$ corresponds to the odd modes of the outer beams. The region $B$ and $C$ correspond to the modes of the central beam and the even modes of the outer beams.

\section{Wavelength Stabilization}

It is possible for the laser to satisfy the following conditions by the use of a suitable diaphragm, and by the adjustment of both the position of the diaphragm and of the excitation energy for the laser medium.

1. The threshold value for the central beam must be low enough to supress the oscillation in the outer beams at the wavelengths almost equal to those of the outer beams.

2. This threshold value must be high enough so that single mode operation is attained when one of the modes is located approximately at the center of the laser line.

3. The outer beams must be kept in single modes, regardless of the change in the cavity spacing at least up to half a wavelength.

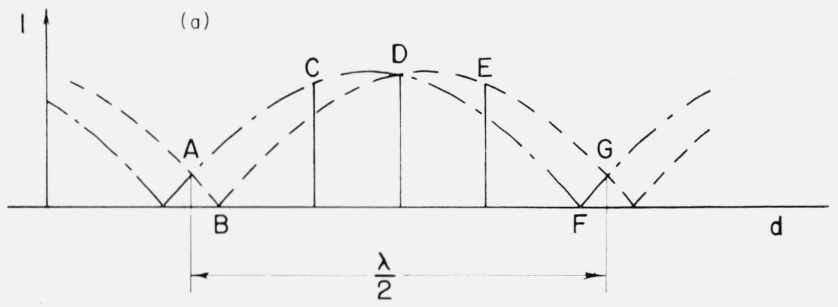

FIgURE 20a. Intensity variation versus cavity spacing.
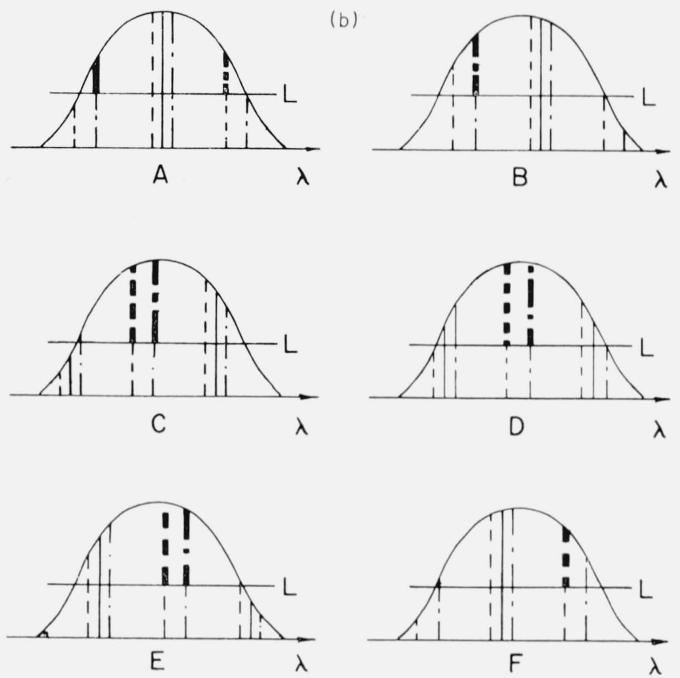

FIgURE 20b. Mode relations at the spacings marked in (a).

4. The intensities of the outer beams must not vanish at the same time.

These conditions are necessary to enable the outer beams to stabilize the wavelength of the central beam.

When the wavelength from a five-beam laser, with each beam in single mode, are located as shown in figure 17, the intensity of the side beams (3) in figure 2 increases and that of the top and bottom beams (2) decreases with a slight increase of the cavity spacing. They will change in a reverse manner with a slight decrease of the spacing. Figure 20a schematically shows the intensity variations with respect to the spacing, $d$.

This variation may be understood from figure $20 \mathrm{~b}$ which shows the relations among the wavelengths, the gain distribution, and the threshold value, $L$, for the outer beams, at various spacings marked in figure 20a. Both intensities become equal at $A$ and $D$. Between $A$ and $D$, one of them is more intense than the other and this relation is reversed in the region between $D$ and $G$. This effect can therefore be used to keep the spacing at $A$ or $G$ by means of a negative feedback. Thus the wavelength of the central beam can be kept approximately at the center of the gain distribution, if the spacing of the cavity is controlled 
to be held at $A$. A phase plate can be inserted within the cavity, instead of making use of aberration in the Brewster's angle windows, in order to produce the mode relation shown in figure $20 \mathrm{~b}$. In this case, it is possible to bring the wavelength to the center of the Doppler distribution with either optical or electronic means. The reproducibility of the wavelength depends mainly upon the intensities of the beams. In this respect, a further experiment is to be carried out.

The author expresses his sincere thanks to K. F. Nefflen for his technical assistance throughout these experiments and for reviewing this paper and to K. D. Mielenz for helpful discussions.

\section{References}

[1] A. Javan, E. A. Ballik, and W. L. Bond, J. Opt. Soc. Am. Letters 52, 96 (1962).

[2] T. S. Jaseja, A. Javan, and C. H. Townes, Phys. Rev Letters 10, 165 (1963).

[3] G. Gould, Frequency 1, 14 (1963).

[4] G. D. Boyd and J. P. Gordon, Bell System Tech. J. 40, 489 (1961).

[5] A. G. Fox and T. Li, Bell System Tech. J. 40, 453 (1961).

[6] W. R. Bennett, Jr., W. L. Faust, and R. A. McFarlane, Their work has been referred to on page 29 in the article by W. R. Bennett, Jr. in Supplement on Optical Masers, Applied Optics (1962).

(Paper 68C1-149) 